\title{
Zur Zusammenarbeit zwischen pädagogischen und nicht-pädagogischen Mitarbeitenden in der Erwachsenenbildung - eine empirische Analyse der Perspektive von Verwaltungskräften
}

\author{
Julia Franz $\cdot$ Markus Scheffel
}

Eingegangen: 13. Januar 2017 / Angenommen: 13. März 2017 / Online publiziert: 30. März 2017 (C) Der/die Autor(en) 2017. Dieser Artikel ist eine Open-Access-Publikation.

Zusammenfassung Die Perspektive des nicht-pädagogischen Personals in pädagogischen Einrichtungen wird in der Forschung der Erwachsenenbildung kaum in den Blick genommen. Auf dieses Forschungsdesiderat reagierend, wird mit diesem Beitrag die Frage verfolgt, wie die Zusammenarbeit von nicht-pädagogischen und pädagogischen Mitarbeitenden in Organisationen der Erwachsenenbildung aus Sicht der Verwaltungskräfte wahrgenommen wird. Diese Frage wird im Rahmen einer qualitativen Analyse bearbeitet, bei der das Datenmaterial einer umfangreicheren Studie genutzt wurde. So wurden Gruppendiskussionen mit Verwaltungskräften dokumentarisch reanalysiert, um die kollektiven Perspektiven auf die Zusammenarbeit in den Organisationen zu rekonstruieren. In der Analyse werden zwei unterschiedliche Muster zur Wahrnehmung der Zusammenarbeit herausgearbeitet, die sich hinsichtlich der Bewertung der Strukturbedingungen der gemeinsamen Kommunikation, des Erlebens der Austauschprozesse und des Erlebens von Hierarchie unterscheiden. Diese Muster werden abschließend im Kontext theoretischer Überlegungen zum professionellen Handeln in Organisationen diskutiert.

Schlüsselwörter Verwaltungskräfte · Erwachsenenpädagogische Organisationen · Zusammenarbeit · Kommunikation

\section{The cooperation of pedagogical and administrational staff in Adult Education - a systematic analyses of the administrational perspective}

Abstract The perspective of administrational staff in educational organizations is
barely in focus in adult education research. This paper reacts on this desideratum of
research and tries to answer the following question: How does the administrational

Prof. Dr. J. Franz $(\bowtie) \cdot$ M. Scheffel

Otto-Friedrich-Universitat Bamberg, Bamberg, Deutschland

E-Mail: Julia.Franz@uni-bamberg.de 
staff of Adult education organizations perceive the collaboration with the pedagogical staff? This question is answered within a qualitative research design. Therefore, the existing data of a qualitative study concerning cultures of teaching in adult education was reanalyzed. In this study, group discussions were conducted with the pedagogical and the administrational staff. According to the research question of this paper the group discussion of administrational staff was reanalyzed in order to reconstruct the collective perspectives of collaboration inside the organizations. The results show two different patterns of perception. They differ in the evaluation of the structural conditions of the organizational communication, the experience of the information sharing process and the experience of hierarchy. Finally these patterns will be discussed in context of theoretical considerations on professionalization in organizations.

Keywords Administrational staff · Adult education organizations · Collaboration · Communication

\section{Einführung}

In Organisationen der Erwachsenenbildung übernehmen pädagogische und nichtpädagogische Mitarbeitende verschiedene Aufgabenbereiche, verfügen über differenzierte Qualifizierungen und sind durch unterschiedliche Anstellungsverhältnisse an die Organisation gebunden. Die Differenz dieser Mitarbeiterformation stellt pädagogische Organisationen potenziell in ein Spannungsfeld zwischen Professionalität ${ }^{1}$ und Organisation (Klatezki und Tacke 2005), da Verwaltung und Pädagogik unterschiedliche Professionalitätslogiken zugrunde liegen. Während aus der Perspektive der Verwaltung Professionalität als Ausdruck der Einhaltung standardisierter, administrativer und formaler Regelungen interpretiert werden kann, kann pädagogische Professionalität als situatives, kompetentes Handeln, dass sich Standardisierungsprozessen entzieht, verstanden werden (auch Hartz 2004). Dabei wird eine Differenz zwischen einer organisations- und einer interaktionsbezogenen Handlungslogik erkennbar. Trotz dieser Differenz arbeiten pädagogische und nicht-pädagogische Mitarbeitende gemeinsam am Organisationzweck, der in der Ermöglichung von Bildungsprozessen besteht. Wie dieses gemeinsame Ziel in der arbeitsteiligen Alltagspraxis gestaltet und wie dabei die Zusammenarbeit zwischen pädagogischen und nicht pädagogischen Mitarbeitenden von Verwaltungskräften erlebt wird, soll in diesem Beitrag durch eine empirische Reanalyse in den Blick genommen werden.

Dazu wird zunächst die Forschungslage zur Zusammenarbeit in pädagogischen Organisationen skizziert, um aufbauend auf dem hier sichtbar werdenden Forschungsdesiderat die Fragestellung der Reanalyse zu präzisieren. Im Anschluss daran wird das methodische Vorgehen dargestellt, bevor die empirischen Ergebnisse

\footnotetext{
1 Während der Begriff „Profession“ ein akademisches Berufsfeld bezeichnet, das u. a. über ein Handlungsmonopol verfügt, beschreibt der Begriff „Professionalisierung“ den „Prozess der Verberuflichung, und zwar auf politischer wie individueller Ebene" (Hartz 2004, S. 239). Der in diesem Beitrag fokussierte Begriff der Professionalität bezieht sich auf das kompetente, situative Handeln im Berufsfeld.
} 
zur Wahrnehmung der Zusammenarbeit aus Sicht der Verwaltungskräfte präsentiert werden. Abschließend werden die Ergebnisse im Spannungsfeld zwischen Organisation und Profession diskutiert.

\section{Zur Zusammenarbeit von pädagogischen und nicht-pädagogischen Mitarbeitenden: die Forschungslage}

Die Zusammenarbeit von pädagogischen und nicht-pädagogischen Mitarbeitenden wurde in der empirischen Forschung bislang kaum untersucht. Dies liegt mitunter daran, dass die unterschiedlichen Akteure im Kontext einer Dichotomisierung von „Organisation“ und „Profession“ aus professionstheoretischen Perspektiven, in denen Organisationen als Rahmenbedingungen der Professionalität aufscheint, isoliert betrachtet werden. Diese isolierte Betrachtung erscheint gerade aufgrund der Entwicklung, dass insbesondere Verwaltungskräfte zunehmend auch pädagogische Aufgaben übernehmen, wie die Betreuung von Kursleitenden und Beratung von Teilnehmenden (Heuer und Gieseke 2006; Dietsche 2006), die Unterstützung von Lehrveranstaltungen sowie die Mitarbeit an Programmplanung und Qualitätsmanagement (von Hippel und Fuchs 2010; von Hippel 2010) unterkomplex.

In der organisationspädagogischen Forschung finden sich zudem Hinweise auf die Bedeutung der organisationalen Zusammenarbeit unterschiedlicher Akteure in pädagogischen Organisationen. In Studien zur Schulkultur (Helsper 2008; Helsper et al. 2001), zum Programmplanungshandeln in Erwachsenenbildungsorganisationen (Gieseke 2003; Dollhausen 2008) oder zum organisationalen Lernen (Feld 2010; Nuissl und Dollhausen 2007; Hittlemann 2007; Dirkx 2006) wird deutlich, dass für die Zusammenarbeit die Verschränkung und Berücksichtigung unterschiedlicher professioneller Sichtweisen von Bedeutung sind, die durch gemeinsame Lernund Entwicklungsprozesse begünstigt werden. In diesen Studien werden allerdings meist die Einstellungen von Leitungen und pädagogischem Personal in den Blick genommen, während nicht-pädagogisches Personal in der Regel unberücksichtigt bleibt. Dies gilt auch für Studien zur Führung pädagogischer Einrichtungen (für einen internationalen Forschungsüberblick siehe von Rosenstil 2011). National wie international wird hier die Bedeutung von kooperativen, dialogischen Leitungsansätzen herausgearbeitet (z. B. Hartley 2010; Tellado 2007; Hunter 2006; Herbrechter 2011). Dabei werden Anerkennung und Wertschätzung als wichtige Komponenten der Mitarbeitermotivation betont (z. B. Rosenbusch 2005). Auch hier werden tendenziell die Perspektiven von Leitungen mit denen von pädagogischen Mitarbeitenden relationiert, während die Perspektive von Verwaltungskräften außen vor gelassen wird.

Dezidierte Hinweise auf die Strukturen der Zusammenarbeit aus Sicht von nichtpädagogischen Mitarbeitenden finden sich vor allem in zwei Untersuchungen.

Erstens: In einer Studie zu Organisationsveränderungen von Monika Kil (2003) wurde unter anderem die Einschätzung des Führungsverhaltens durch Verwaltungskräfte mit untersucht. In der quantitativen Analyse von Verwaltungsmitarbeitenden in nordrheinwestfälischen Volkshochschulen wird deutlich, dass diese Mitarbeitergruppe eine geringe Wertschätzung empfindet und sich diese negativ auf ihre Motivation 
und das Klima der Organisation auswirkt (Kil 2003, S. 104). Darüber hinaus werden in dieser Studie die Arbeitsbedingungen von nicht-pädagogischem Verwaltungspersonal in den Blick genommen. Hier zeigt sich, dass Verwaltungsmitarbeitende im Vergleich zu anderen Mitarbeitenden das niedrigste Motivierungspotenzial aufweisen (ebd., S. 113). Dabei konnten verschiedene Typen der Verarbeitung dieses geringen Potentials gruppiert werden (de-motiviert, ambivalent-motiviert, konstruktivmotiviert und stabil-motiviert). Konstruktiv-motivierte Muster fänden sich vor allem bei Verwaltungsmitarbeitenden, die viele Freiräume im eigenen Handeln erleben würden.

Zweitens: In einer qualitativ explorativen Studie zu den Perspektiven auf Verwaltung in Organisationen der Erwachsenenbildung (Dietsche 2015) wurde zudem untersucht, was unterschiedliche Mitarbeitende unter ,Verwaltung“ verstehen und wie sie die Relation zwischen Verwaltung und Pädagogik konzipieren. Dabei wurde deutlich, dass Verwaltung aus drei Blickwinkeln betrachtet wird: Zunächst kann Verwaltung als Handlung - wie zum Beispiel als Dokumentieren oder Organisieren - verstanden werden (ebd., S. 192). Zum zweiten zeigt sich eine institutionalisierte Sicht, die sich auf die spezifische Abteilungsform von Verwaltung bezieht (ebd., S. 56). Zum dritten wird mit Verwaltung eine jeweils unterschiedliche Sinnperspektive verknüpft, die mit induktiv hergeleiteten Zuschreibungen wie ,Verwaltung ist Unterstützung“ (ebd., S. 63) oder „Verwaltung hat Macht inne“ (ebd., S. 64) belegt wird. Darüber hinaus zeigt die Studie, dass Verwaltungstätigkeiten eine Schlüsselrolle bei der Vermittlung zwischen konkretem Kursgeschehen, der Weiterbildungseinrichtung und Trägern einnehmen (ebd., S. 165). Schließlich wird mit der Studie darauf verwiesen, dass Verwaltung und Pädagogik als Berufspositionen verstanden werden, die in der täglichen Arbeit aufeinander bezogen sind. In diesem Zusammenhang werden die beschriebene Vermengungen von pädagogischen und verwaltenden Handlungen als „Mischtätigkeiten“ gefasst (ebd., S. 194).

Gerade in den letztgenannten Studien wird deutlich, dass Verwaltung und Pädagogik zwar als getrennte Berufspositionen verstanden werden, diese aber im Hinblick auf die Aufgaben pädagogischer Organisationen - wie Programmplanung und Kursumsetzung - aufeinander bezogen werden müssen. Zu der Frage, wie diese Zusammenarbeit von den Verwaltungskräften wahrgenommen wird und welche Kommunikationsprozesse sich im Prozess der Verknüpfung beider Tätigkeiten abspielen, gibt es bislang keine empirischen Erkenntnisse. An dieses Desiderat wird mit diesem Beitrag angeschlossen und danach gefragt, wie die Zusammenarbeit in erwachsenenpädagogischen Organisationen von Verwaltungskräften wahrgenommen wird.

\section{Methodisches Vorgehen}

Diese Forschungsfrage wird im Rahmen einer Reanalyse des Datenmaterials einer Studie zum Lehren in Organisationen Allgemeiner Erwachsenenbildung (Franz 2016a) bearbeitet. ${ }^{2}$

\footnotetext{
${ }^{2}$ Für die Förderung der Forschungsarbeit danken wir der Deutschen Forschungsgemeinschaft (Förderzeichen: FR 2716/2-2).
} 


\subsection{Zur Datengrundlage}

In der qualitativ-rekonstruktiven Studie zu den „Kulturen des Lehrens“ in Organisationen Allgemeiner Erwachsenenbildung (Franz 2016a) wurden in einem theoretischen Samplingprozess (Glaser und Strauss 1967) neun Organisationen einbezogen. Es handelt sich dabei um Einrichtungen, die vor allem ein breites Spektrum allgemeinbildender und politischer Inhalte anbieten. In das Sample wurden Einrichtungen konfessioneller, zivilgesellschaftlicher öffentlicher Trägerschaft integriert.

In der Datenerhebung dieser Studie wurden jeweils Interviews mit den Leitungspersonen sowie separate Gruppendiskussionen (Loos und Schäffer 2001) mit hauptamtlich pädagogischen Mitarbeitenden, Kursleitenden und Verwaltungskräften geführt. Insgesamt liegen 23 Gruppendiskussionen und neun Leitungsinterviews vor. Die Auswertung der Daten erfolgte durch die dokumentarische Methode (Bohnsack et al. 2007) und führte zu einer mehrdimensionalen Typenbildung (Franz 2016a).

Durch die offenen Erhebungsmethoden konnten im vielschichtigen Datenmaterial weitere Fragestellungen identifiziert werden, die im Kontext einer Anschlussstudie bearbeitet werden. Neben Fragen zum Einfluss regionaler, räumlicher Situierungen der Organisationen (Franz 2016b) wird es durch das mehrperspektivische Datenmaterial und die getrennte offene Erhebung der Perspektive von Verwaltungskräften und pädagogischen Mitarbeitenden auch möglich, die Kommunikationsstrukturen in deren Zusammenarbeit in den Blick zu nehmen. Da in den jeweiligen Eingangsimpulsen vor allem angeregt wurde, über das Lehren und Lernen in der Einrichtung zu sprechen, erscheint es nicht verwunderlich, dass die pädagogischen Mitarbeitenden vor allem (mikro)didaktische Perspektiven entfalten. Sie kommen selbstläufig nicht auf Strukturen der Zusammenarbeit in der Organisation zu sprechen. Die Verwaltungskräfte betonen hingegen, eher für die Rahmenbedingungen des Lehren und Lernens zuständig zu sein und erzählen selbstläufig von den Kommunikationsstrukturen, den Herausforderungen und den Grenzen in der Zusammenarbeit mit den pädagogischen Mitarbeitenden. Vor diesem Hintergrund wurde in der Reanalyse die - ohnehin unterbeforschte - Perspektive der Verwaltungskräfte auf die Zusammenarbeit untersucht. ${ }^{3}$

\subsection{Methodisches Vorgehen der Reanalyse}

Für die Forschungsfrage nach der Wahrnehmung der Zusammenarbeit wurden daher die Gruppendiskussionen mit den Verwaltungskräften reanalysiert. Diese wurden nach der dokumentarischen Methode ausgewertet (Bohnsack et al. 2007). Mit dieser Methode wird es durch die komparative Analyse und die formulierenden und reflek-

\footnotetext{
3 An den Gruppendiskussionen haben in der Regel alle angestellten Verwaltungskräfte teilgenommen. Sie wurden jeweils von den Leitungen der Einrichtung gebeten, an diesen Termin anwesend zu sein. Nach einer anfänglichen Gesprächsunsicherheit aufgrund der Annahme, nicht viel über das Lehren erzählen zu können, entwickelten sich allerdings selbstläufige Gespräche, an denen sich alle Verwaltungskräfte beteiligten. Die Diskussionen dauerten jeweils zwischen ein und zwei Stunden. Die Berufsausbildung der befragten Verwaltungskräfte ist recht heterogenen. Eine Mehrzahl verfügt jedoch über Ausbildungen im kaufmännischen Bereich. Im gesamten Sample gibt es nur eine Verwaltungskraft, die angibt, an einer Fortbildung zur organisationspädagogischen Mitarbeitenden teilzunehmen.
} 
tierenden Interpretationsschritte möglich, implizite Wissensbestände von expliziten zu unterscheiden (Mannheim 1980) und darüber insbesondere die handlungsleitenden Orientierungen zu rekonstruieren. Entsprechend wurde in der Reanalyse darauf abgezielt, die impliziten und kollektiv geteilten Perspektiven von Verwaltungskräften zur Zusammenarbeit in Organisationen Allgemeiner Erwachsenenbildung zu rekonstruieren. Die Analyse bezieht sich daher nicht auf die Individualperspektiven der Akteure, sondern auf die Rekonstruktion kollektiver Wissensstrukturen von Verwaltungskräften in Organisationen. Konkret wurden die Passagen im Material formulierend und reflektierend interpretiert, in denen die Verwaltungskräfte auf Aspekte der Zusammenarbeit innerhalb ihrer Organisation eingegangen sind.

\section{Empirische Ergebnisse - Muster der Wahrnehmung von Zusammenarbeit}

In der Reanalyse wird zunächst deutlich, dass in den Gruppendiskussionen mit Verwaltungskräften die Zusammenarbeit im Hinblick auf drei zentrale Aspekte diskutiert wird bzw. implizit relevant wird. Dabei handelt es sich um die Strukturbedingungen der kommunikativen Zusammenarbeit, um die Funktion von Austauschprozessen und um das Erleben von Hierarchie. Dabei werden jeweils unterschiedliche Wertungen dieser Themen sichtbar. Im Folgenden werden zunächst die drei Aspekte, die in allen Gruppendiskussion zum Thema werden, deskriptiv dargestellt, bevor im Anschluss die unterschiedlichen Bewertungen zu zwei Mustern ${ }^{4}$ der Zusammenarbeit verdichtet werden - Zusammenarbeit als problematische Kommunikation im Modus eines steilen Hierarchieerlebens (4.1) und Zusammenarbeit als kommunikative Transparenz im Modus eines flachen Hierarchieerlebens (4.2).

Strukturbedingungen der kommunikativen Zusammenarbeit. Zum einen wird im empirischen Material deutlich, dass die Verwaltungskräfte unterschiedliche Arbeitszeiten von hauptamtlich pädagogischen Mitarbeitenden, Leitungen und Verwaltungskräften thematisieren. Während vor allem die Pädagoginnen und Pädagogen beispielsweise durch Abend- oder Wochenendveranstaltung unregelmäßige Arbeitszeiten hätten, hätten die Verwaltungskräfte - die häufig in Teilzeit tätig seien regelmäßige Arbeitszeiten. Vor diesem Hintergrund wird die Bedeutung von ,überlappenden Arbeitszeiten“ (Organisation A) betont, in den Absprachen gemeinsam getroffen werden müssten. Die unterschiedlichen Arbeitszeiten werden damit zu einer Strukturbedingung in der kommunikativen Zusammenarbeit, die im empirischen Material von den Verwaltungskräften unterschiedlich bewertet werden. In einigen Organisationen problematisieren Verwaltungskräfte die Konsequenzen für die Gestaltung von Austauschprozessen, während in anderen Organisationen diese Bedingungen als Selbstverständlichkeit betrachtet werden.

\footnotetext{
${ }^{4}$ Bei diesen Mustern handelt es sich - methodologisch betrachtet - um eine idealtypische Verdichtung, die einer sinngenetischen Typenbildung vorgeschaltet ist.
} 
Zur Funktion von Austauschprozessen: Zum zweiten werden im Material unterschiedliche Möglichkeiten des Austausches zwischen pädagogischen und nichtpädagogischen Mitarbeitenden beschrieben. Diese reichen von informellen Gesprächen über „offene Türen“(Organisation G, F) über formalisierte Teambesprechungen (Organisation A, B, D, E, F, G, H, I) bis zu formalen, schriftlichen Dokumentationen, wie z. B. Protokollen (Organisation H). In der Beschreibung all dieser Kommunikationsformen zeigt sich die Funktion von Austauschprozessen aus Sicht der Verwaltungskräfte. Ihre Aufgabe liegt vor allem in der Kommunikation nach außen. Sie müssen mit (potenziellen) Teilnehmenden ebenso wie mit Kooperationspartnern, Kursleitenden und Personen aus den Trägerverbänden oder der kommunalen Öffentlichkeit kommunizieren. Vor diesem Hintergrund betonen alle Verwaltungskräfte, dass es wichtig sei, ,,aussagefähig“ zu sein. Die Austauschprozesse mit den Pädagoginnen und Pädagogen haben daher für die Verwaltungskräfte die Funktion, Informationen und Hintergrundwissen zu erhalten, um ihre eigene Berufsrolle funktional erfüllen zu können. Auch hier werden im Material zwei unterschiedliche Bewertungen deutlich. In einigen Organisationen werden Austauschprozesse als transparent und funktional beschrieben, während in anderen Organisationen Informationslücken bemängelt werden und der Austausch eher als dysfunktional wahrgenommen wird.

Zum Erleben von Hierarchie in der Kommunikation: Schließlich wird im Material implizit deutlich, dass für die Zusammenarbeit zwischen Verwaltungskräften und Pädagoginnen und Pädagogen das Erleben von Hierarchie eine zentrale Rolle spielt. So zeigt sich im Material, das die Differenz der Berufsbereiche grundsätzlich hierarchisch interpretiert wird. ${ }^{5}$ Im Material lassen sich hier wieder zwei unterschiedliche Bewertungen rekonstruieren. In einigen Organisationen wird Hierarchie als ,flache Hierarchie“ im Sinne einer horizontalen Arbeitsteilung interpretiert, während in anderen Organisationen Verwaltungskräfte Hierarchie eher als ,steile Hierarchie“, mit der auch Abwertungen der eigenen Rolle verbunden sind, erleben.

Aus den unterschiedlichen Bewertungen dieser drei zentralen Themen lassen sich aus dem empirischen Material heraus zwei idealtypische Muster zur Wahrnehmung der Zusammenarbeit von Verwaltungskräften und pädagogischen Mitarbeitenden verdichten, die im Folgenden vorgestellt werden.

\subsection{Zusammenarbeit als problematische Kommunikation im Modus eines steilen Hierarchieerlebens}

Das erste Muster zeichnet sich dadurch aus, dass von den Verwaltungskräften vor allem Kommunikationsprobleme und dysfunktionale Austauschprozesse beschrieben werden. Zudem werden Hierarchien als steil interpretiert und als über- und untergeordnete Positionen in den Einrichtungen verstanden. Vor diesem Hintergrund werden die drei identifizierten Hauptthemen wie folgt bewertet.

\footnotetext{
5 Besonders eindrücklich zeigt sich dies darin, dass in der Beschreibung der „offenen Türen“ davon gesprochen wird, dass die Türen gelegentlich von den Pädagoginnen und Pädagogen geschlossen werden (Organisation F), während die Praktik des Türe Schließens von den Verwaltungskräften nicht getätigt wird.
} 
Kommunikationsprobleme als Konsequenz der Strukturbedingung der kommunikativen Zusammenarbeit: Das Muster ,Zusammenarbeit als problematische Kommunikation im Modus eines steilen Hierarchieerlebens“ zeichnet sich dadurch aus, dass die unterschiedlichen Arbeitszeiten zwischen pädagogischen und nicht-pädagogischen Mitarbeitenden problematisiert werden. So wird beispielsweise in Organisation B ein daraus resultierendes Kommunikationsproblem thematisiert (Abb. 1).

Im in Abb. 1 dargestellten Ausschnitt wird eine mangelnde Ansprechbarkeit der pädagogischen Mitarbeitenden thematisiert. Implizit wird den Pädagogen auch ein schlechtes Zeitmanagement unterstellt, dass dazu führt, dass diese sich auf die Verwaltungskräfte verlassen. Die Verwaltungskräfte sehen sich in der Rolle, für die Pädagogen ,mitdenken“ (Organisation B, Z. 135) zu müssen, da die Erwartung, dass „die Pädagogen eigentlich auf uns zukommen sollten“ (Organisation B, Z. 137 f.) nicht erfüllt werde. Dahinter deutet sich an, dass die Zusammenarbeit durch Verwaltungskräfte initiiert werden muss, dies aber gleichwohl negativ wahrgenommen wird. Schon hier deutet sich anhand der beschriebenen Kommunikationsprobleme an, dass die Arbeitsbereiche von Verwaltung und Pädagogik als nicht gleichberechtigt wahrgenommen werden.

$\mathrm{Zu}$ dysfunktionalen Austauschprozessen: Zum zweiten wird in diesem Muster sichtbar, dass auch die Informationsaustauschprozesse problematisiert werden. Es wird beschrieben, dass diese aus Sicht der Verwaltungskräfte nicht hinreichend gut gelingen, um aussagekräftig zu sein. Die eigene Berufsaufgabe, adäquat nach außen zu kommunizieren wird so erschwert. Dies zeigt sich beispielsweise beim Ausschnitt in Organisation A. (Abb. 2).

Im Transkriptauschnitt, der in Abb. 2 dargestellt wird, zeigt sich, dass die Verwaltungskräfte Informationslücken haben und ihnen das Hintergrundwissen fehlt, um klar nach außen kommunizieren zu können. Die offenbar mangelnde Transparenz in internen Austauschprozessen führt für diese Verwaltungskräfte zu einem negativen Erleben der eigenen Position, in der sie wie ein „Dummchen“ da stehen würden. Es

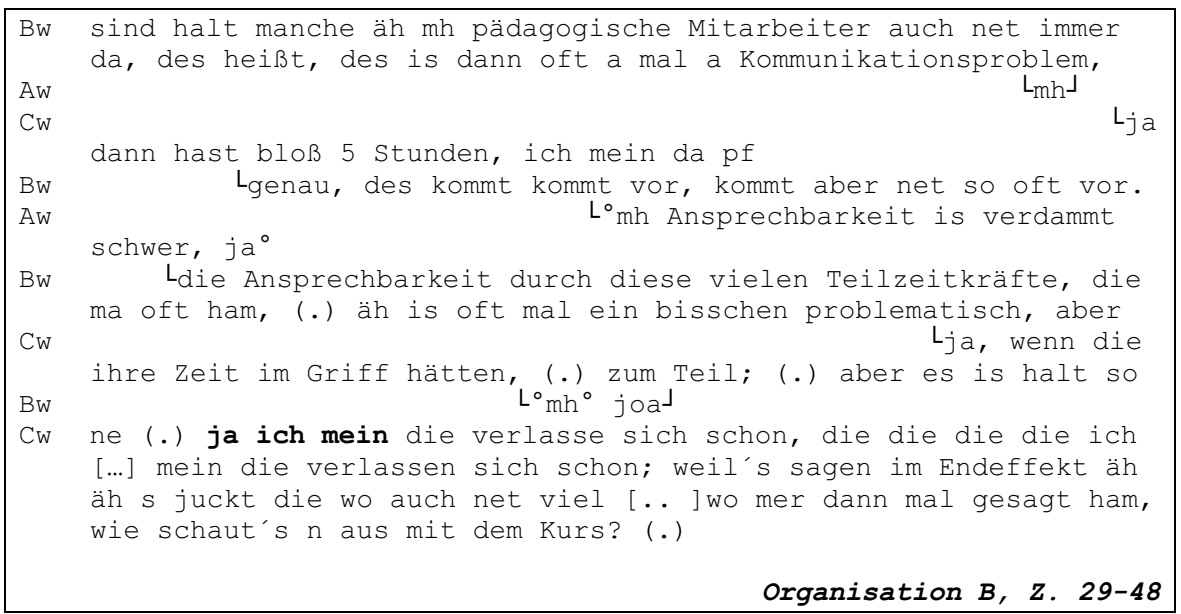

Abb. 1 Transkriptausschnitt, Organisation B 


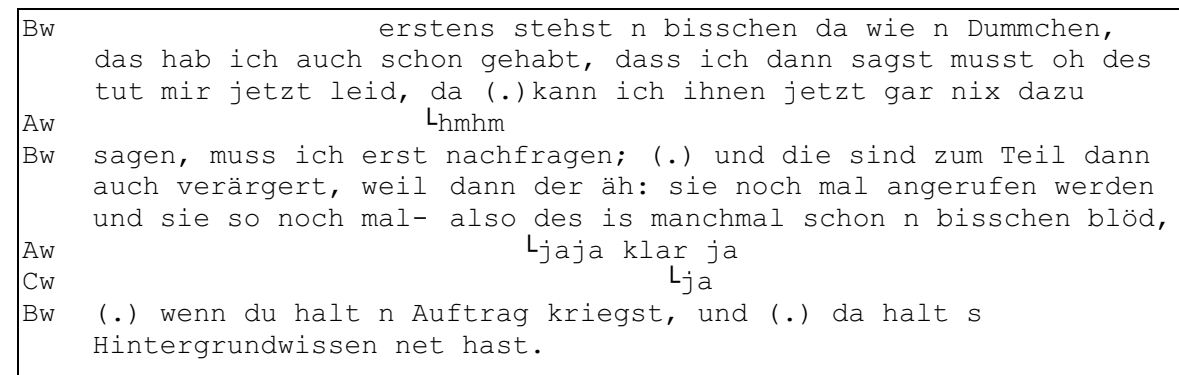

Organisation A, Z. 328-341

Abb. 2 Transkriptausschnitt, Organisation A

zeigt sich, dass sich die Verwaltungskräfte hier nicht ausreichend informiert fühlen und dies ihre alltägliche Arbeit erschwert bzw. belastet.

Steiles Hierarchieerleben: Zum dritten wird hinsichtlich des Hierarchieerlebens deutlich, dass Verwaltungskräfte in diesem Muster Hierarchie als steil interpretieren und damit unterschiedliche Positionen in der Organisation verknüpfen. Dabei nehmen die Verwaltungskräfte die niedrige Position ein und empfinden sich teilweise den höheren Positionen ausgeliefert. Beispielsweise wird von den Verwaltungskräften aus Organisation A beschrieben, dass die pädagogischen Mitarbeitenden nicht „,konflikt- und kommunikationsfähig“ (Organisation A; Z. 67 f.) seien, dies aber gerade von ihnen als Verwaltungskräften erwartet werden würde. Darin deutet sich an, dass die Verwaltungskräfte sich hier in einer untergeordneten Position sehen, die sie sich qua Amt, nicht jedoch qua Kompetenz zuschreiben. Diese erlebte Amtshierarchie führt in Organisation A auch dazu, dass ,,man sich selber oft gar nicht als Team empfindet“" (Organisation A, Z. 82 ff.). In anderen Organisationen zeigt sich ein solches Hierarchieerleben auch darüber, dass man sich beispielsweise mit Leitungen und Vorstandsmitgliedern ganz unkompliziert unterhalten könne (Organisation I). Darin wird deutlich, dass aufgrund der Hierarchie hier davon ausgegangen wird, dass dies nicht selbstverständlich, sondern eine Besonderheit darstellt. Zusammenfassend lässt sich sagen, dass bei diesem Muster Hierarchie von den Verwaltungskräften negativ erlebt wird und sie aus ihrer Sicht eine den pädagogischen Mitarbeitenden untergeordnete Position in Organisationen einnehmen.

\subsection{Zusammenarbeit als kommunikative Transparenz im Modus eines flachen Hierarchieerlebens}

Das zweite Muster zeichnet sich dadurch aus, dass die Bedeutung von gelingender Kommunikation hervorgehoben wird, dass Transparenz eine zentrale Perspektive für die Verwaltungskräfte darstellt und schließlich, dass Hierarchie als flach im Sinne einer Arbeitsteilung wahrgenommen wird.

Selbstverständliches Mitdenken als Konsequenz der Strukturbedingung der kommunikativen Zusammenarbeit: Das Muster der Zusammenarbeit als kommunikative Transparenz im Modus eines flachen Hierarchieerlebens aus der Perspektive der Verwaltungskräfte zeichnet sich dadurch aus, dass die unterschiedlichen Arbeitszeiten 


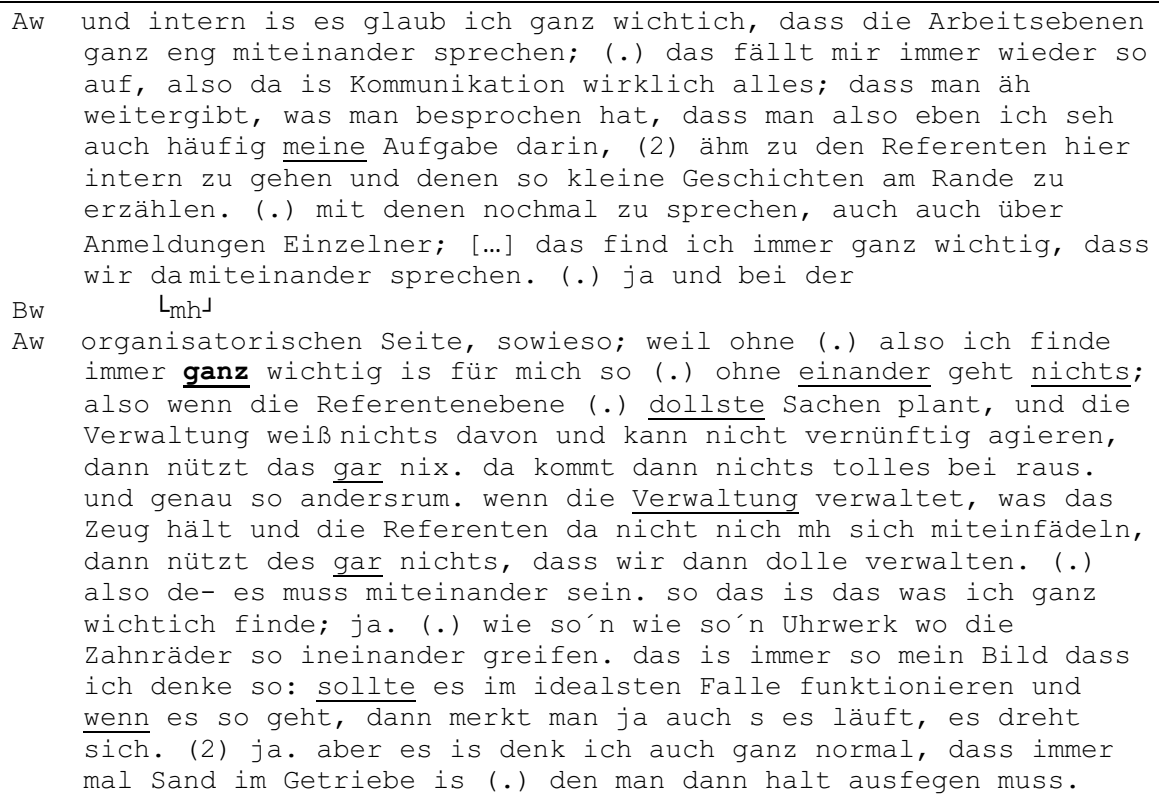

Organisation D; Z. 3-40

Abb. 3 Transkriptausschnitt, Organisation D

der Mitarbeitenden als Selbstverständlichkeit akzeptiert werden. Die Konsequenz für die Verwaltungskräfte besteht darin, die Kommunikation mit den Pädagoginnen und Pädagogen zu suchen und zu organisieren, wie sich aus Transkriptausschnitt in Abb. 3 herauslesen lässt.

Im in Abb. 3 dargestellten Ausschnitt wird betont, dass es Aufgabe der Verwaltungskräfte sei, auf die Referenten zuzugehen und „Geschichten am Rande“ zu erzählen. Dahinter dokumentiert sich ein inkludierendes Organisationsverständnis, bei dem Verwaltung und Pädagogik als „Zahnräder eines Uhrwerks“ interpretiert werden, bei dem die Handlungen ineinandergreifen. Beide Bereiche werden als gleichwertig und als untrennbar miteinander verwoben interpretiert. Vor diesem Hintergrund erscheint für diese Verwaltungskräfte Kommunikation als selbstverständliche Notwendigkeit.

Transparenz als Ergebnis funktionaler Austauschprozesse: Zum zweiten wird in diesem Muster die Funktion von Austauschprozessen, mit dem Ziel aussagekräftig zu sein, sehr positiv eingeschätzt. Es wird beschrieben, dass über informelle Gespräche, Protokolle oder Teambesprechungen eine große Transparenz hergestellt werde. Dies zeigt sich beispielsweise im Ausschnitt der Verwaltungskräfte aus Organisation G. (Abb. 4).

Im Ausschnitt, der in Abb. 4 dargestellt wird, zeigt sich, dass die Dienstberatungen dazu dienen, Transparenz über Geschehnisse herzustellen und über die Termine aller Mitarbeitenden zu informieren. Damit ist die Funktion, die die Verwaltungskräfte ihrer Tätigkeit zuschreiben, eng verbunden. Es geht vor allem darum, aussagekräftig 


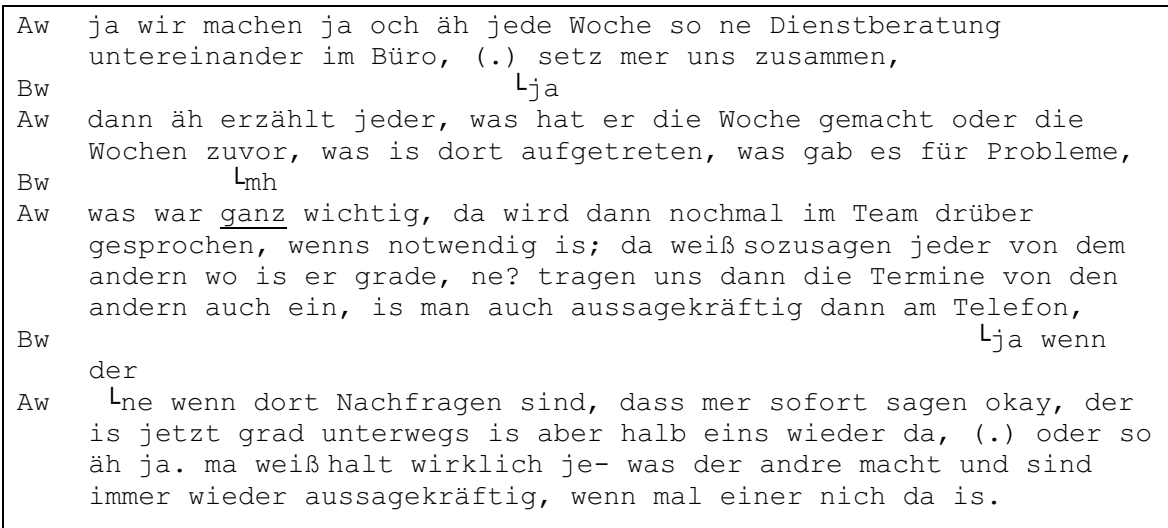

Organisation G, Z. 1043-1058

Abb. 4 Transkriptausschnitt, Organisation G

in der Kommunikation nach außen zu sein. Um diese Funktion erfüllen zu können bedarf es - wie im Ausschnitt sichtbar wird - vor allem interner Transparenz. Da die Pädagogen und Pädagoginnen nicht immer im Hause sind, erscheint es für die Verwaltungskräfte zentral, genau über deren An- und Abwesenheiten und Aufenthaltsorte Bescheid zu wissen. Darüber hinaus geht es aber auch inhaltlich in den Dienstbesprechungen um „Probleme“, die in der Woche aufgetreten sind. Charakteristisch für dieses Muster erscheint daher, dass die Bedeutung von Transparenz betont und diese gleichzeitig für die eigene Organisation als sehr hoch eingestuft wird.

Flaches Hierarchieerleben: Schließlich wird in diesem Muster Hierarchie in der Zusammenarbeit als flach - im Sinne einer gleichberechtigten Arbeitsteilung - wahrgenommen. Typisch dafür erscheinen beispielsweise Aussagen über das selbstverständliche Mitspracherecht bei wichtigen Entscheidungen, wie ein Ausschnitt aus Organisation E zeigt (Abb. 5).

Im in Abb. 5 dargestellten Transkriptausschnitt wird deutlich, dass das Mitspracherecht im Hinblick auf „Beschlüsse“ von den Verwaltungskräften wertgeschätzt wird. Die Betonung der gemeinschaftlichen Entscheidungen im Team verweist darauf, dass die Verwaltungskräfte sich als Teil dieses Teams und - im Gegensatz zum ersten Muster - nicht in Abgrenzung dazu positionieren. Diese Vergemeinschaftungsperspektive wird auch an der Verwendung der Formulierung der ,,großen Familie“ deutlich. Wie auch in anderen Organisationen sichtbar wird, führen Partizipationsmöglichkeiten und Spielräume im eigenen Verwaltungshandeln dazu, sich als Teil eines großen Ganzen (Organisation E; D) oder als Familie (Organisation E, D) zu verstehen, bei den ,,alle am gleichen Strang ziehen“ (Organisation G; Z. 1289) und alle das gleiche Ziel hätten. Hierarchie wird in diesem Muster daher als flach und als Ausdruck einer bereichsspezifischen und arbeitsteilig organisierten Expertise verstanden. Es geht also weniger um eine Über- oder Unterordnung der Berufsgruppen, sondern um ein gleichberechtigtes Nebeneinander. 


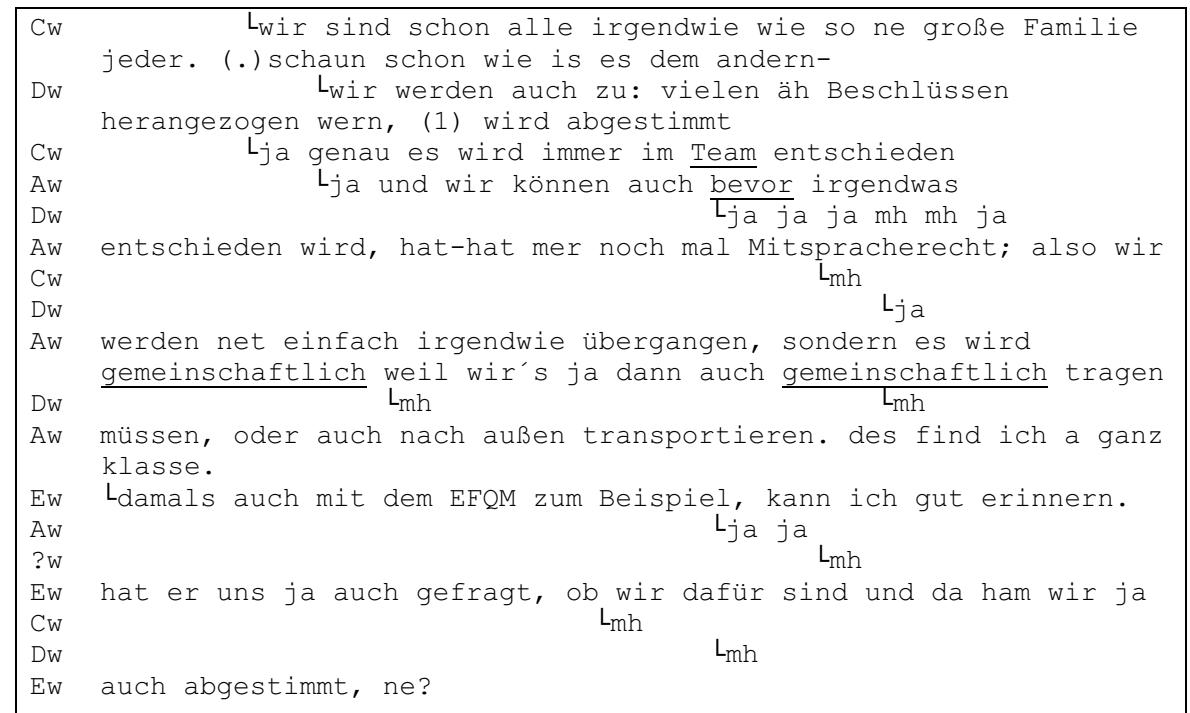

Organisation E, Z. 501-522

Abb. 5 Transkriptausschnitt, Organisation E

\subsection{Zusammenfassung}

Zusammenfassend lässt sich sagen, dass die rekonstruierten Muster jeweils auf der unterschiedlichen Bewertung der zentralen Themen (Strukturbedingungen der kommunikativen Zusammenarbeit, Erleben von Austauschprozessen und Erleben von Hierarchie) aufbauen (Tab. 1).

Beide Muster liefern Anregungen dazu, wie auch professionelles Handeln von unterschiedlichen Berufsgruppen in pädagogischen Organisationen betrachtet werden kann. Dieser Aspekt wird in der abschließenden Diskussion in den Blick genommen.

Tab. 1 Die Muster zur Wahrnehmung zur Zusammenarbeit im Überblick

\begin{tabular}{|c|c|c|c|c|}
\hline Muster & Bewertung & Themen & Bewertung & Muster \\
\hline \multirow[t]{3}{*}{$\begin{array}{l}\text { Zusammenarbeit } \\
\text { als kommunikative } \\
\text { Transparenz im } \\
\text { Modus eines } \\
\text { flachen } \\
\text { Hierarchieerlebens }\end{array}$} & $\begin{array}{l}\text { Selbstverständ- } \\
\text { lichkeit }\end{array}$ & $\begin{array}{l}\text { Unterschiedliche } \\
\text { Arbeitszeiten } \\
\text { als Strukturbe- } \\
\text { dingungen der } \\
\text { kommunikativen } \\
\text { Zusammenarbeit }\end{array}$ & Problematisierung & $\begin{array}{l}\text { Zusammenarbeit } \\
\text { als problematische } \\
\text { Kommunikation } \\
\text { im Modus eines } \\
\text { steilen } \\
\text { Hierarchieerlebens }\end{array}$ \\
\hline & Funktional & $\begin{array}{l}\text { Erleben von Aus- } \\
\text { tauschprozessen }\end{array}$ & Dysfunktional & \\
\hline & Flach & $\begin{array}{l}\text { Erleben von } \\
\text { Hierarchie }\end{array}$ & Steil & \\
\hline
\end{tabular}




\section{Diskussion: Zur Zusammenarbeit zwischen Profession und Organisation}

Die Ergebnisse der empirischen Reanalyse zeigen, dass die Zusammenarbeit zwischen pädagogischen und nicht-pädagogischen Mitarbeitenden in erwachsenenpädagogischen Organisationen aus Sicht der Verwaltungskräfte im Kontext zweier idealtypischer Wahrnehmungsmuster betrachtet wird. ${ }^{6}$ Beide Muster sollen abschließend im Spannungsfeld zwischen Profession und Organisation diskutiert werden. Schließlich liefern beide Muster Hinweise dazu, wie die Zusammenarbeit von unterschiedlichen Berufsgruppen im Kontext von professionellem Handeln verstanden werden kann.

So verweist in erster Linie das Muster „Zusammenarbeit als kommunikative Transparenz im Modus eines flachen Hierarchieerlebens" auf die Bedeutung des Konzepts einer so genannten ,organisationsgebundenen Professionalität“ (Schicke 2012). Dieses Konzept fußt auf einer im Diskurs beobachtbaren Annäherung zwischen Organisationstheorie und Professionstheorie (z. B. Vanderstraeten 2008; Seitter 2011, S. 133), mit denen Organisation und Profession (Klatetzki und Tacke 2005) nicht mehr gegenübergestellt, sondern vielmehr als zwei Formen beschrieben werden, über die sich das Erziehungssystem ausdifferenziert (Kurtz 2004, S. 45). Professionalität wird hier jenseits der Dualität von Struktur und Handlung betrachtet: „Professionalität konstituiert sich wechselseitig, denn Akteure/Akteurinnen beziehen sich reflexiv und rekursiv auf die pädagogischen Institutionalformen, die sie immer wieder situativ zu den Modalitäten ihres Handelns machen“ (Schicke 2012, S. 70).

Interessant erscheint nun, dass unsere Befunde deutlich machen, dass diese organisationsgebundene Professionalität nicht nur zur Betrachtung der einzelnen Berufsgruppen innerhalb einer Organisation dienen kann, sondern möglicherweise auch dazu dient, das Zusammenspiel ineinandergreifender Handlungen (Weick 1995) zwischen Pädagogik und Verwaltung in pädagogischen Organisationen als Ausdruck einer organisationsgebundenen Professionalität zu betrachten. Organisationen, in denen beide Bereiche von den Verwaltungskräften als gleichwertig betrachtet werden, haben damit das Potenzial, eine übergeordnete Form professionellen Handelns zu entwickeln, das auf den Prämissen transparenter Kommunikationsstrukturen basiert. Die Differenz organisations- und interaktionsbezogener Handlungslogiken wird somit anerkannt und in der Kommunikation bearbeitet. Im Gegensatz dazu verweist das Muster ,Zusammenarbeit als problematische Kommunikation im Modus steilen Hierarchieerlebens" darauf, dass eine solche übergeordnete kommunikative Professionalität unterschiedlicher Berufsgruppen nicht entsteht, wenn die Berufsgruppen im Modus der Unter- und Überordnung eingeteilt werden. Hier wird die Differenz zwischen organisations- und interaktionsbezogener professioneller Handlungslogik hierarchisiert. Diese Hierarchisierung führt tendenziell zu einem niedrigen Motivierungspotenzial (Kil 2003, S. 104) von Verwaltungskräften, das die Zusammenarbeit in den Organisationen beeinflusst. Für eine übergeordnete kommunikative organi-

\footnotetext{
${ }^{6}$ Es handelt sich hier um eine idealtypische Verdichtung. Natürlich ist davon auszugehen, dass in der Praxis durchaus ambivalente Mischformen existieren (die allerdings im Material nicht eindeutig rekonstruiert werden konnten).
} 
sationsgebundene Professionalität, in der die Differenz zwischen Interaktion und Organisation bearbeitet werden kann - so die aus den Ergebnissen abgeleitete These -, erscheint also vor allem das Erleben von Hierarchie zentral.

In diesem Zusammenhang bietet es sich für die künftige Forschung an, den Umgang mit Hierarchie zwischen pädagogischen und nicht pädagogischen Mitarbeitenden genauer in den Blick zu nehmen. Besonders interessant wäre es beispielsweise im Kontext von Organisationsentwicklungsprozessen zu untersuchen, auf welche Art und Weise unterschiedliche Mitarbeitendengruppen eingebunden werden. Für die Praxis wird zudem impliziert, dass die Einbindung in zentrale Entscheidungen und die wertschätzende und transparente Kommunikation bedeutsam erscheint, um eine übergeordnete kommunikative Professionalität in Organisationen zu ermöglichen.

Open Access Dieser Artikel wird unter der Creative Commons Namensnennung 4.0 International Lizenz (http://creativecommons.org/licenses/by/4.0/deed.de) veröffentlicht, welche die Nutzung, Vervielfältigung, Bearbeitung, Verbreitung und Wiedergabe in jeglichem Medium und Format erlaubt, sofern Sie den/die ursprünglichen Autor(en) und die Quelle ordnungsgemäß nennen, einen Link zur Creative Commons Lizenz beifügen und angeben, ob Änderungen vorgenommen wurden.

\section{Literatur}

Bohnsack, R., Nentwig-Gesemann, I., \& Nohl, A.-M. (Hrsg.). (2007). Die dokumentarische Methode und ihre Forschungspraxis. Grundlagen qualitativer Sozialforschung (2. Aufl.). Wiesbaden: Springer VS.

Dietsche, B. (2006). Fortbildung für Verwaltungsmitarbeitende in Weiterbildungseinrichtungen. In U. Heuer \& W. Gieseke (Hrsg.), Pädagogisches Wissen für die Weiterbildung. Fortbildungsbedarf und Personalentwicklung (S. 185-227). Oldenburg: Textweinberg.

Dietsche, B. (2015). Verwaltung in Weiterbildungseinrichtungen. Vom diffusen Unbehagen zum professionell-reflektierten Umgang mit Verwaltungstätigkeiten. Wiesbaden: Springer VS.

Dirkx, J.M. (2006). „Singing to the choir:“ The struggle for systemic organizational change in community college teaching. In Proceedings of the annual Adult Education Research Conference. http://www. adulterc.org/Proceedings/2006/Proceedings/DirkxConner.pdf. Zugegriffen: 08. Jan 2017.

Dollhausen, K., \& Nuissl, E. (Hrsg.). (2007). Bildungseinrichtungen als ,,lernende Organisationen“? Befunde aus der Weiterbildung. Wiesbaden: Springer VS.

Dollhausen, K. (2008). Planungskulturen in der Weiterbildung. Angebotsplanungen zwischen wirtschaftlichen Erfordernissen und pädagogischem Anspruch. Bielefeld: W. Bertelsmann.

Feld, T.C. (2010). Entwicklung von Organisationen als organisationspädagogische Gestaltungsaufgabe. Anforderungsprofil an lernende Weiterbildungsorganisationen. Zeitschrift für Erziehungswissenschaft ZfE, 13(1), 83-98.

Franz, J. (2016a). Kulturen des Lehrens. Eine Studie zu kollektiven Lehrorientierungen in Organisationen Allgemeiner Erwachsenenbildung. Bielefeld: W. Bertelsmann.

Franz, J. (2016b). Zur Bedeutung von „Raum“ in Organisationen Allgemeiner Erwachsenenbildung - Ergebnisse einer qualitativ-rekonstruktiven Studie. Zeitschrift für Erziehungswissenschaft ZfE, 19(1), 31-53.

Gieseke, W. (2003). Programmplanungshandeln als Angleichungshandeln: Die realisierte Vernetzung in der Abstimmung von Angebot und Nachfrage. In W. Gieseke (Hrsg.), Institutionelle Innensichten der Weiterbildung (S. 189-211). Bielefeld: W. Bertelsmann.

Gieseke, W. (2006). Programmforschung als Grundlage der Programmplanung unter flexiblen institutionellen Kontexten. In K. Meisel \& C. Schiersmann (Hrsg.), Zukunftsfeld Weiterbildung. Standortbestimmungen für Forschung, Praxis und Politik (S. 69-88). Bielefeld: W. Bertelsmann.

Glaser, B. G., \& Strauss, A. L. (1967). The discovery of grounded theory: strategies for qualitative research. New York: Adline.

Hartley, D. (2010). The management of education and the social theory of the firm: from distributed to collaborative community. Journal of Educational Administration and History, 42(4), 345-362. 
Hartz, S. (2004). Qualität in der Weiterbildung: die Perspektivengebundenheit von Qualitätsanforderungen am Beispiel der Differenz von Organisation und Profession. In W. Fröhlich \& W. Jütte (Hrsg.), Qualitätsentwicklung in der postgradualen Weiterbildung. Internationale Entwicklungen und Perspektiven (S. 231-248). Krems: Edition Donau-Universität Krems.

Helsper, W., Busse, S., Hummrich, M., \& Kramer, R. T. (Hrsg.). (2001). Pädagogische Professionalität in Organisation. Neue Verhältnisbestimmungen am Beispiel der Schule. Wiesbaden: Springer VS.

Helsper, W. (2008). Schulkulturen als symbolische Sinnordnungen und ihre Bedeutung für pädagogische Professionalität. In W. Helsper, S. Busse, M. Hummrich \& R.-T. Kramer (Hrsg.), Pädagogische Professionalität in Organisationen. Neue Verhältnisbestimmungen am Beispiel der Schule (S. 115-145). Wiesbaden: Springer VS.

Herbrechter, D. (2011). Leitung und Wissen in Weiterbildungsorganisationen. Befunde einer empirischen Analyse. In M. Göhlich, S. M. Weber, Chr. Schiersmann \& A. Schröer (Hrsg.), Organisation und Führung (S. 77-86). Wiesbaden: Springer VS.

Heuer, U., \& Gieseke, W. (Hrsg.). (2006). Pädagogisches Wissen für die Weiterbildung, Fortbildungsbedarf und Personalentwicklung. Oldenburg: Textweinberg.

von Hippel, A., \& Fuchs, S. (2010). Herausforderungen für das berufliche Handeln von Erwachsenenbildner/innen. Eine Analyse aus unterschiedlichen Perspektiven. In C. Hof, J. Ludwig \& B. Schäffer (Hrsg.), Erwachsenenbildung im demographischen und sozialen Wandel (S. 52-68). Baltmannsweiler: Schneider Verlag Hohengehren.

von Hippel, A. (2010). Erwachsenenbildner/innen an der Schnittstelle zwischen Verwaltung und Pädagogik. Eine explorative Analyse der Tätigkeitsfelder von Verwaltungsmitarbeiter/inne/n mit pädagogischen Aufgaben. Report - Zeitschrift für Weiterbildungsforschung ZfW, 33(2), 77-88.

Hittleman, M. J. (2007). When do we stop asking, why"? Exploring the possibilities and challenges of collective critical reflection for staff and organizational development. In proceedings of the Annual Adult Education Research Conference. http://www.adulterc.org/Proceedings/2007/Proceedings/Hittleman. pdf. Zugegriffen: 8. Jan. 2017.

Hunter, S. (2006). Working for equality and diversity in adult and community learning: leadership, representation and racialised outsiders within. Policy Futures in Education, 4(2), 114-127.

Kil, M. (2003). Organisationsveränderungen in Weiterbildungseinrichtungen. Empirische Analysen und Ansatzpunkte für Entwicklung und Forschung. Bielefeld: W. Bertelsmann.

Klatetzki, T., \& Tacke, V. (Hrsg.). (2005). Organisation und Profession. Wiesbaden: Springer VS.

Kurtz, T. (2004). Organisation und Profession im Erziehungssystem. In W. Böttcher (Hrsg.), Organisationstheorie in pädagogischen Feldern. Analyse und Gestaltung (S. 43-53). Wiesbaden: Springer VS.

Loos, P., \& Schäffer, B. (2001). Das Gruppendiskussionsverfahren. Theoretische Grundlagen und empirische Anwendung. Opladen: Leske + Budrich.

Mannheim, K. (1980). Strukturen des Denkens. Frankfurt a.M.: Suhrkamp.

Rosenbusch, H. S. (2005). Organisationspädagogik der Schule. Grundlagen pädagogischen Führungshandelns. München: Luchterhand.

Von Rosenstiel, L. (2011). Führung in Organisationen - Facetten eines Konzepts, Wirkmechanismen, Erfolgskriterien. In M. Göhlich, S.M. Weber, Chr. Schiersmann \& A. Schröer (Hrsg.), Organisation und Führung (S. 27-43). Wiesbaden: Springer VS.

Schicke, H. (2012). Organisation als Kontext der Professionalität. Beruflichkeit pädagogischer Arbeit in der Transformationsgesellschaft. Bielefeld: W. Bertelsmann.

Seitter, W. (2011). Wandel des Professionellen im Feld der Weiterbildung. In W. Helsper \& R. Tippelt (Hrsg.), Pädagogische Professionalität (S. 122-137). Weinheim: Beltz.

Tellado, I. (2007). Dialogic management in adult education. In proceedings of the Annual Adult Education Research Conference. http://www.adulterc.org/Proceedings/2007/Proceedings/Tellado.pdf. Zugegriffen: 08. Jan 2017.

Vanderstraeten, R. (2008). Zwischen Profession und Organisation. Professionsbildung im Erziehunsgsystem. In W. Helsper, S. Busse, M. Hummrich \& R.-T. Kramer (Hrsg.), Pädagogische Professionalität in Organisationen. Neue Verhältnisbestimmungen am Beispiel der Schule (S. 9-113). Wiesbaden: Springer VS.

Weick, K. E. (1995). Der Prozess des Organisierens. Berlin: Suhrkamp. 